\title{
Neutrophil Expression of T and B Immunomodulatory Molecules in HIV Infection
}

\section{OPEN ACCESS}

Edited by:

Gabriella d'Ettorre,

Sapienza University of Rome, Italy

Reviewed by:

Yolanda María Pacheco, Institute of Biomedicine of Seville

(CSIC), Spain

Camilla Tincati,

University of Milan, Italy

*Correspondence: José-Antonio Girón-González

joseantonio.giron@uca.es

${ }^{+}$These authors have contributed equally to this work and share last authorship

Specialty section: This article was submitted to Microbial Immunology, a section of the journa

Frontiers in Immunology

Received: 22 February 2021 Accepted: 29 November 2021 Published: 17 December 2021

Citation:

Márquez-Coello M Ruiz-Sánchez C, Martín-Aspas A, Fernández Gutiérrez Del Álamo C, Illanes-Álvarez F, Cuesta-Sancho S and Girón-González J-A (2021)

Neutrophil Expression of

$T$ and $B$ Immunomodulatory

Molecules in HIV Infection.

Front. Immunol. 12:670966. doi: 10.3389/fimmu.2021.670966
Mercedes Márquez-Coello ${ }^{1,2}$, Cristina Ruiz-Sánchez ${ }^{1,2}$, Andrés Martín-Aspas ${ }^{1,2}$, Clotilde Fernández Gutiérrez Del Álamo ${ }^{2,3}$, Francisco Illanes-Álvarez ${ }^{1,2}$, Sara Cuesta-Sancho ${ }^{1,2 t}$ and José-Antonio Girón-González ${ }^{1,2 *}$

1 Unidad de Enfermedades Infecciosas, Servicio de Medicina Interna, Hospital Universitario Puerta del Mar, Facultad de Medicina, Universidad de Cádiz, Cádiz, Spain, 2 Instituto de Investigación e Innovación en Ciencias Biomédicas de Cádiz (INiBICA), Cádiz, Spain, ${ }^{3}$ Servicio de Microbiología, Hospital Universitario Puerta del Mar, Facultad de Medicina, Universidad de Cádiz, Cádiz, Spain

Objective: Evaluate the expression of $\mathrm{B}$ and $\mathrm{T}$ cell immunomodulatory molecules in polymorphonuclear neutrophils (PMN) in HIV-infected patients.

Methods: HIV load, bacterial translocation and neutrophils' expression of T [programmed death ligand, interleukin-10+, arginase 1+] and B [BAFF, APRIL] molecules were analyzed in different cohorts and time points: a control group of 25 healthy individuals and two groups of HIV-infected patients. Group 1 of patients included 35 untreated patients, studied at baseline and after antiretroviral therapy (ART). Group 2 was composed of 25 patients with undetectable viral load after a median of 101 months of ART prior to inclusion in the study.

Results: Compared with the control group, group 1 patients showed increased bacterial translocation and their PMN had a significantly higher expression of $\mathrm{T}$ and $\mathrm{B}$-cell immunomodulatory molecules, both at baseline and after 12 months of ART. Group 2 patients showed reduced bacterial translocation levels when compared with group 1 patients after 12 months of treatment. PMN expression of B-cell modulators was similar between group 2 patients and healthy controls, although the expression of T-cell modulators remained increased.

Conclusion: In HIV-infected patients, the expression of B-cell stimulatory and T-cell suppressive molecules by neutrophils was increased at baseline and after a limited time of therapy. After a prolonged period of ART, only PMNs expression of T-cell immunosuppressive molecules remained elevated.

Keywords: HIV, neutrophils, BAFF, APRIL, bacterial translocation, interleukins, arginase-1, PDL-1 


\section{INTRODUCTION}

Polymorphonuclear neutrophils (PMNs) have been considered to be effector elements of the antimicrobial immune response (1). PMNs recognize microbes through pathogen-associated molecular patterns, including Toll-like receptors (TLRs). Activation of TLRs in PMNs induces the production of proinflammatory cytokines, expression of adhesion molecules and activation of reactive oxygen species (ROS) (2).

The influence of PMNs on adaptive immunity has gained relevance in recent years. In acute bacterial infection, PMNs synthesize interleukin (IL)-12, provoking $\mathrm{T}$ cell differentiation in $\mathrm{T}$ helper 1 (Th1) cells. Under other circumstances, such as during chronic viral infections, PMNs are able to suppress Tlymphocyte activation (3). The neutrophil-dependent $\mathrm{T}$ cell down-regulation is mediated by the synthesis of arginase-1 (4), IL-10 production (5), programmed death antigen-1 ligand (PDL1) overexpression (6) and transformation in myeloid-derived suppressor cells (7).

PMNs also synthesize B lymphocyte activating factors, such as the B cell-activating factor of the tumor necrosis factor family (BAFF) and a proliferation-inducing ligand (APRIL) (8). After binding to their receptors on B cells, BAFF and APRIL promote the survival and differentiation of B cells amongst plasma cells, stimulating the synthesis of immunoglobulin $M$ (IgM) and a switch to IgG or IgA (8). Microbes stimulate BAFF and APRIL expression in PMNs, macrophages and dendritic cells, either by binding to TLRs or indirectly via interferon I and II synthesis (9).

After acute infection by the human immunodeficiency virus (HIV), a massive depletion of $\mathrm{CD} 4+\mathrm{T}$ cells in gut-associated lymphoid tissues occurs and persists throughout the chronic stage (10). The administration of antiretroviral therapy (ART) only partially repairs gut mucosal injury $(10,11)$. Due to the CD4+ T cell loss and intestinal barrier damage, microbial translocation occurs in HIV-infected patients (12) showing an increase in serum levels of lipopolysaccharide and 16S ribosomal DNA (16S rDNA), which are markers of this bacterial translocation (12).

It is theoretically possible that $\mathrm{T}$ or B-cell immunomodulatory molecules in PMNs could be induced in HIV-infected patients either by HIV itself, via TLR7/8 interaction (13), or by bacterialderived products secondary to bacterial translocation, either via TLR4-bacterial lipopolysaccharide or TLR9-16S rDNA interactions (2). Attending to this hypothesis, only a limited information about expression of T-cell inhibitory molecules (arginase-1, PDL-1) by PMNs has been reached in HIV-infected individuals $(14,15)$. The analysis of the expression of B-cell immunomodulatory molecules by PMNs has not been performed.

The objective of this study is to perform a quantitative evaluation of the expression of $\mathrm{T}$ and $\mathrm{B}$ cell modulatory molecules in PMNs in HIV-infected patients a) before ART, a situation in which both stimuli, HIV itself and microbialtranslocation-derived products, could be operative, b) after 12 months of treatment, when gut barrier damage and bacterial translocation persist but HIV replication is controlled, and c) in a sample of HIV-infected individuals that had undergone more than 8 years (101 months) of therapy, to evaluate the long-term consequences of viral suppression on the expression of these molecules in PMNs.

\section{PATIENTS AND METHODS}

\section{Study Design}

A prospective, observational study of consecutive cases of HIV infection recruited from a cohort of patients followed up in an HIV outpatient clinic at the Puerta del Mar University Hospital (Cádiz, Spain) was conducted.

$60 \mathrm{HIV}$-infected adults were divided in the following groups: 1) Group 1 included 35 naïve patients. They were analyzed at the time of inclusion in the study and after 6 and 12 months with ART. 2) Group 2 included 25 patients with chronic HIV infection and ART-induced undetectable HIV viral loads at inclusion [undetectable HIV loads for a median of 101 months (range, 60-154 months)]. Comparison of results of PMNs derived molecules between group 1 patients after 12 months of treatment (when undetectable HIV viral load had been obtained) and group 2 individuals (with a longer period of undetectability) was performed. Twenty-five age- and gender-matched healthy individuals were recruited from voluntary hospital workers as healthy controls.

The exclusion criteria were as follows: 1) clinical records suggesting acute HIV infection; 2) active or other opportunistic infections [including viral hepatitis, Pneumocytis jirovecii, toxoplasmosis, tuberculosis, cytomegalovirus infections, etc] or neoplasms. Our screening procedure followed the guidelines established by the Spanish Group for AIDS Study (www.gesida.seimc.org); 3) active drug use (cocaine, heroin, amphetamines) or significant alcohol ingestion (greater than 50 g/day); 4) treatments that could have modified the determination of inflammation-related molecules or cells (pentoxifylline, anti-inflammatory or immunosuppressive drugs); 5) red blood cell or plasma transfusion in the month before inclusion; and 6) antibiotic treatment, which might modify the intestinal flora.

HIV infection was diagnosed using an chemiluminescent microparticle immunoassay (Alinity HIV Ag/Ab Combo assay, Ludwigshafen, Germany) for the simultaneous qualitative detection of HIV p24 antigen and antibodies to HIV type 1 and type 2 in serum. Positive results were confirmed by detection of HIV load (Abbott RealTime HIV-1, Abbott Park, IL, USA).

The duration of the HIV infection was established based on the first positive anti-HIV test.

When the HIV load was less than 50 copies/ml (Abbott RealTime HIV-1, Abbott Park, IL, USA), HIV replication was considered to be controlled.

Increased serum concentration of intestinal fatty acid-binding protein (I-FABP) was indicative of gut barrier disruption (16). The bacterial translocation was detected by serum $16 \mathrm{~S}$ rDNA levels (17).

\section{Study Schedule}

The protocol included: 1) clinical history, nadir $\mathrm{CD} 4+\mathrm{T}$ cell count, and time with undetectable HIV load;2) CD4+ T cell 
count, CD4+/CD8+ T cells ratio, and HIV RNA levels at inclusion in the study; 3) blood count, biochemistry, and coagulation determinations by automated procedures; 4) peripheral blood sampling at inclusion for analysis of bacterial translocation, immune and inflammatory parameters.

In patients with detectable HIV loads at baseline (Group 1), ART was initiated and followed for 12 months according to the Spanish Group for AIDS Study guidelines [https://gesida-seimc. org]. Their immune parameters were analyzed every six months.

Current ART was continued in those patients with undetectable HIV loads at inclusion (group 2) and we only determined their immune parameters at inclusion.

\section{Laboratory Methods Sample Collection}

Serum was obtained by centrifugation at $1600 \mathrm{~g}$ during $15 \mathrm{~min}$ at room temperature from blood samples in pyrogen-free heparinized tubes (Biofreeze, Costar, USA) and subsequently frozen down at $-80^{\circ} \mathrm{C}$ until its use.

\section{S rDNA Quantification}

16S rDNA was detected after an initial extraction of DNA (QIAamp DNA Mini Kit; QIAgen, Hilden, Germany) and quantification by spectrophotometry (BioRad, Hercules, CA, USA). 16S rDNA region of $E$. coli was amplified using the following primers: $16 \mathrm{~S}$ R 5'-ACC-GCC-ACT-GCT-GCT-GGCAC-3' and 16S F 5'-AGA-GTT-TGA-TCA-TGG-CTG-AG-3' (IDT, Coralville, Iowa, USA). A polymerase chain reaction (PCR) was performed with a standard curve using an E. coli colony for quantification in a CFX Connect Real-Time PCR System (CFX Connect Real-Time PCR System, Bio-Rad Laboratories, Inc. CA, USA). The standard curve of E. coli was obtained from a colony, provided by the Microbiology Service of the Puerta del Mar University Hospital. After heat shock $\left(90^{\circ} \mathrm{C}\right.$ for $10 \mathrm{~min}$ and $10^{\circ} \mathrm{C}$ for $10 \mathrm{~min}$ ) and centrifugation, the supernatant was collected and the concentration was measured by spectrophotometry. After serial dilutions of the colony sample, the RT PCR was carried out together with 3 samples per group of patients/controls to verify that the concentrations of the latter are within the curve obtained. The entire process was carried out in a biological safety horizontal flow hood. An exclusive pipette set for 16S PCR and filter tips were used. RT PCR was carried out on 8-well strips. As a precaution, every strip was covered and stored at $4^{\circ} \mathrm{C}$ when loaded. Finally, the samples were centrifuged and run in CFX.

\section{ELISAS}

Quantikine Human Immunoassays ( $\& \& D$, Minneapolis, $M N$, USA) were used to quantify serum concentrations of I-FABP, IL6 and IL-10. Immunoturbidometry was used to analyze serum immunoglobulin concentrations.

\section{Flow Cytometry}

Fresh cells from whole lysed blood were fixed and stained with blue-fluorescent reactive dye (Live/Dead Fixable Dead Cell Stain Kit; Invitrogen, Carlsbad, CA, USA) to exclude dead cells from analysis. Then, cell suspensions were stained for surface markers, and subsequently, after permeabilization with the Intra Stain (Dako, Denmark A/S), for intracellular molecules. Mouse isotype control conjugates with PE, PerCP, FITC and APC were used to confirm the specificity of the staining and to discriminate the sample from the background.

Stained cells were washed, acquired and analyzed in a FACSCalibur cytometer, using CellQuest software (BD Bioscience, San Jose, CA, USA). In each case, 100000 cells were acquired.

Granulocyte populations were gated based on forward (FSC-H) and side (SSC-H) scatter. In this gate, CD15+ and CD14- cells were selected. For PMN characterization, anti-CD16 (clone VEP13, Miltenyi Biotec, Bergisch, Gladbach, Germany), anti-CD15 (clone W6D3, Becton Dickinson, San Jose, CA, USA), and anti-CD14 (clone TET2, Becton Dickinson) were used (Supplementary Figure 1).

Membrane expression of CD11b (clone ICRF44, Becton Dickinson) and PDL-1 (clone 29E.2A3, Becton Dickinson) were analyzed. Intracellular expression of molecules using antiIL-6 (clone 1936, R\&D), anti-IL-10 (clone JES3-9D7, Milteny Biotec), anti $\mathrm{h} / \mathrm{m}$ arginase-1 (Polyclonal sheep IgG, R\&D Systems, Minneapolis, USA), anti-human CD257 (BAFF, BLYS) (clone T7-241, Biolegend, San Diego, CA, USA) and anti-APRIL (clone A3D8, Biolegend) were determined (Supplementary Figures 2, 3). PMNs molecule expression is indicated as median fluorescence intensity (MFI).

To avoid nonspecific staining and/or antibody binding to nontarget intracellular proteins or neutrophil Fc receptors (18), the following actions were taken: 1) To ensure that the antibodies did not give false positive signals because of the cross-react with intracellular proteins or binding to non-specifically to FcyRII/ CD32 and FcyIII/CD16, cells were treated with a FcR blocking reagent (Miltenyi Biotec). 2) In our protocol, a negative control was always run only with blood, but processed as if it had antibodies. Therefore, when results were analyzed, the signal in the tube with no antibodies was our negative control (cell autoflorescence).

Lymphocytes were gated based on forward (FSC-H) and side (SSC-H) scatter for determination of CD4+ (clone SK3) and CD8+ (clone SK1) populations. Anti CD19 (clone HIB 19, Becton Dickinson, San Jose, CA, USA), anti CD27 (clone M-T271, Becton Dickinson, San Jose, CA, USA) and anti IgD (clone IA6-2, Becton Dickinson, San Jose, CA, USA) were used for B lymphocytes.

\section{Statistical Analysis}

Data were expressed as absolute numbers (percentage) or as median values (25-75 interquartile range (IQR). Categorical variables were compared using the chi-square test or Fisher's exact test. The MannWhitney U test or ANOVA was used to compare quantitative variables from two independent groups. For comparison of the three or more independent groups, Kruskal-Wallis test was used. Friedman' or Wilcoxon's rank tests were used to perform paired analysis of variables. The association between quantitative variables was analyzed using the Spearman correlation test. A two-tailed $\mathrm{p}$ value of $<0.05$ was considered to be significant. SPSS 22.0 statistical software package (SPSS Inc., Chicago, IL, USA) was used to perform statistical analysis. 


\section{Ethical Aspects}

This study was performed according to the Helsinki Declaration. The ethical research committee of Hospital Puerta del Mar (Cádiz, Spain) approved the project. Each participant gave written informed consent.

\section{RESULTS}

The haematological, immune and virological characteristics of untreated patients (Group 1) and controls are shown in Table 1. $71 \%$ of those patients $(n=25)$ showed a CD4+ T cell count lower than $500 / \mathrm{mm}^{3}$.

Serum I-FABP and 16S rDNA concentrations were significantly higher in untreated patients than in controls (Table 2), and a significant correlation between I-FABP concentration and $16 \mathrm{~S}$ rDNA levels $(\mathrm{r}=0.306, \mathrm{p}=0.026)$ was detected.

Serum IL-6 and IL-10 concentrations were significantly higher in untreated HIV-infected individuals than in controls (Table 2).

\section{Activation Level of PMNs in Untreated HIV-Infected Patients}

The activation level of PMNs was measured based on the membrane expression of $\mathrm{CD} 11 \mathrm{~b}$ and the intracellular expression of IL-6. The MFI of CD11b and intracytoplasmic IL-6 were significantly increased in Group 1 patients (Table 2 and Supplementary Figure 2).

\section{PMNs Expression of T and B Cell Modulatory Molecules in Untreated HIV-Infected Patients}

Membrane expression of PDL-1 and intracytoplasmic expression of IL-10 and arginase- 1 as T cell modulatory molecules in PMNs were analyzed, showing an increase in untreated HIV-infected patients (Table 2 and Supplementary Figure 2).

The intracellular expression of BAFF and APRIL in PMNs was significantly higher in untreated HIV-infected individuals (Table 2 and Supplementary Figure 3).

No significant differences between activation parameters or expression of $\mathrm{T}$ or $\mathrm{B}$ modulatory molecules in PMNs were observed between patients with more or less than 500 T CD4+ cells $/ \mathrm{mm}^{3}$ (data not shown).

\section{Evolution of Gut Barrier Alteration, Bacterial Translocation and Immune Markers in Group 1 Patients After ART}

All Group 1 patients started ART and achieved undetectable HIV loads at 6 months. Patients were treated either with tenofovir

TABLE 1 | Hematological, immune and virological characteristics and gut barrier lesion and bacterial translocation markers of healthy controls and HIV-infected patients.

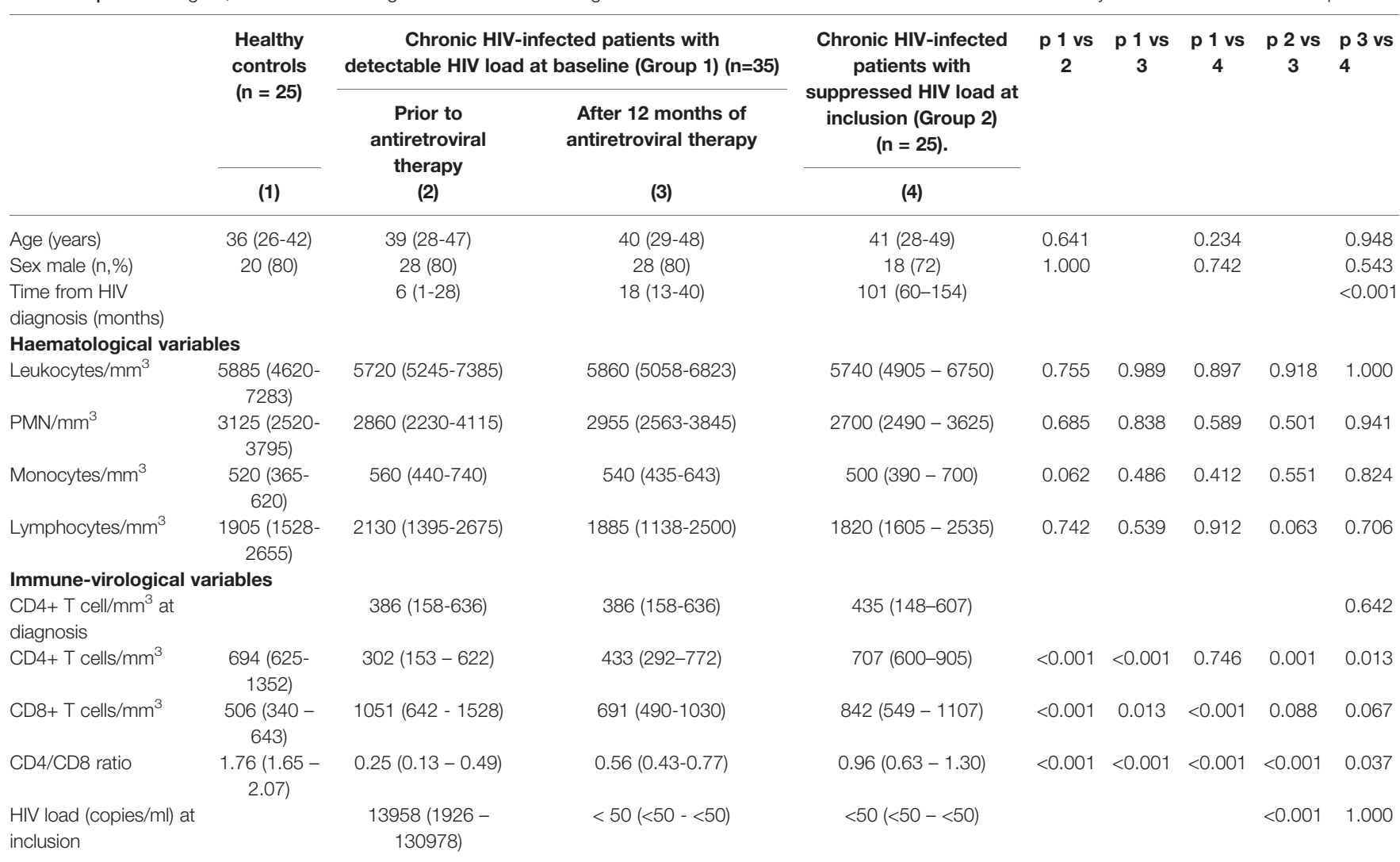

HIV, Human immunodeficiency virus; PMN, Polymorphonuclear

Data are provided as absolute number (percentage) or as median (interquartile range). 
TABLE 2 | Gut barrier status, bacterial translocation and immune characteristics of healthy controls, patients with chronic infection, untreated at baseline, analyzed after 12 months of antiretroviral therapy, and of chronically treated HIV patients with undetectable HIV load at inclusion.

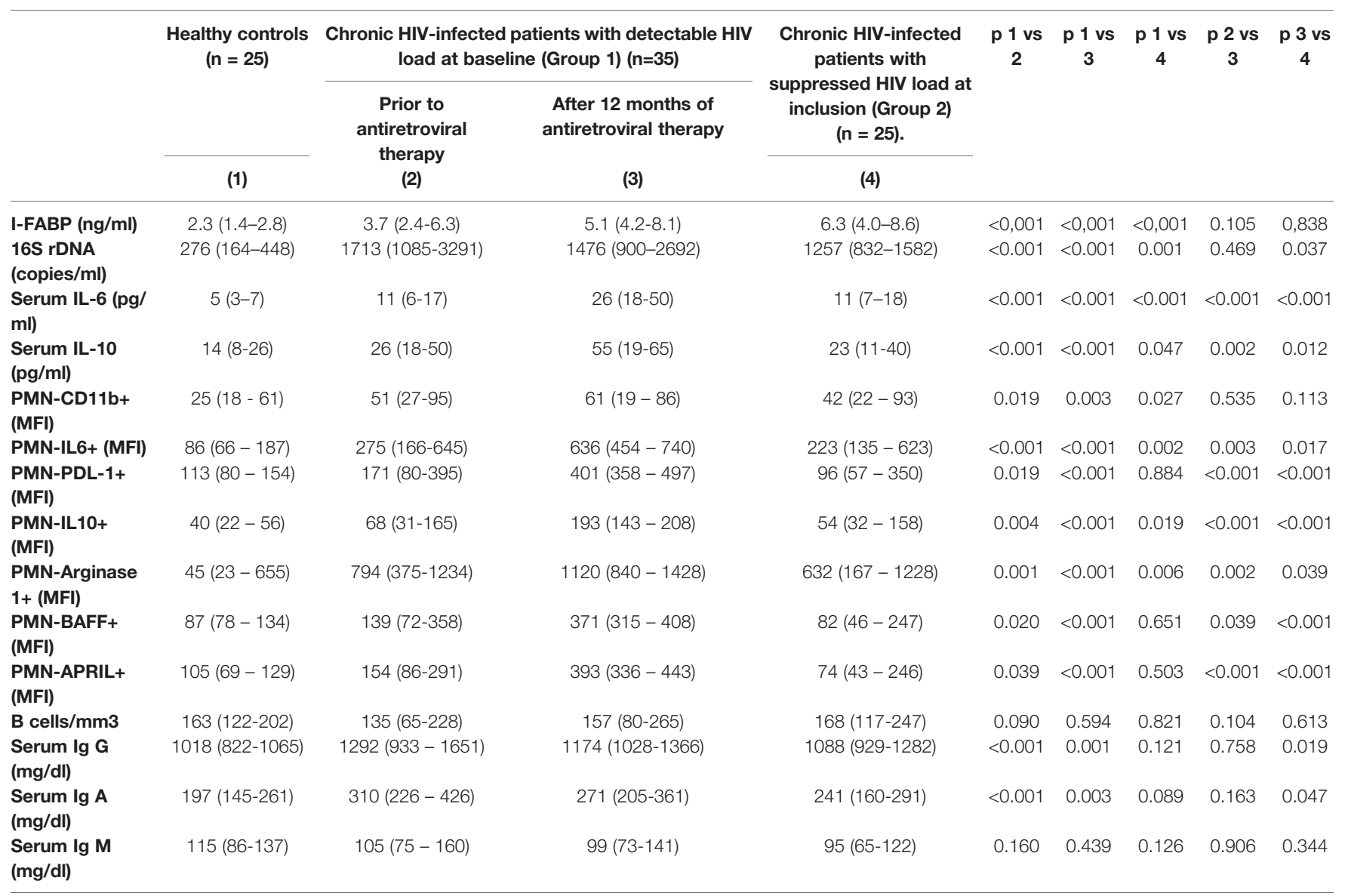

HIV, Human immunodeficiency virus; I-FABP, Intestinal fatty acid binding protein; IL-6, interleukin 6; IL-10, Interleukin 10; PMN, Polymorphonuclear;

MFI, median fluorescence intensity; Ig, Immunoglobulin.

Data are provided as median (interquartile range).

alafenamide and emtricitabine (31 cases) or abacavir and lamivudine (4 cases) associated with integrase inhibitors (23 cases), darunavir/cobicistat (7 individuals) or rilpivirine (5 patients). A significant increase in the CD4+ $\mathrm{T}$ cell count in patients was observed (Table 1). No significant differences were found in B cell counts or IgG, IgA or IgM serum concentrations between data collected at baseline and after 12 months of therapy (Table 2).

During the follow-up, I-FABP concentrations remained elevated, without significant differences when compared with baseline values (Table 2). Likewise, $16 \mathrm{~S}$ rDNA continued to be elevated despite ART (Table 2).

The MFI of IL-6 and T (PDL-1, Arginase-1, IL-10) and B (BAFF and APRIL) modulatory molecules expressed in PMNs had increased significantly after 6-month of ART when compared with the baseline values. Differences between values of MFI obtained after 6 and 12 months of follow-up were nonsignificant (Figures 1 and 2).

IL- 6 serum concentrations increased significantly during the follow-up [baseline, 11 (6-17) pg/ml; after 6 months of ART, 25 (16-34) $\mathrm{pg} / \mathrm{ml}, \mathrm{p}<0.001$ when compared with baseline; after 12 months of ART, $26(18-50) \mathrm{pg} / \mathrm{ml}, \mathrm{p}<0.001$ when compared with baseline; $\mathrm{p}=0.270$ when compared with 6 months of ART]. Also, IL-10 serum concentrations increased significantly during the follow-up [baseline, 25 (18-50) pg/ml; after 6 months of ART, 55 (38-69) pg/ml, p=0.001 when compared with baseline; after 12 months of ART, 55 (19-65) pg/ml, p=0.002 when compared with baseline; $\mathrm{p}=0.100$ when compared with 6 months of ART].

\section{Comparison of PMNs Expression of T- and B-Immunomodulatory Molecules in Naïve HIV-Infected Patients (Group 1) After ART During 12 Months and Patients With Chronically Undetectable HIV Load (Group 2)}

Characteristics observed after 12 months of ART in the $35 \mathrm{HIV}$ infected individuals that had been untreated at baseline (Group 1) were compared with those of 25 chronically infected patients with undetectable HIV at inclusion as a consequence of previous ART (Group 2) [undetectable HIV load for a median of 101 (range, 41-317) months] (Table 1). Group 2 individuals had been treated either with tenofovir alafenamide and emtricitabine 
A

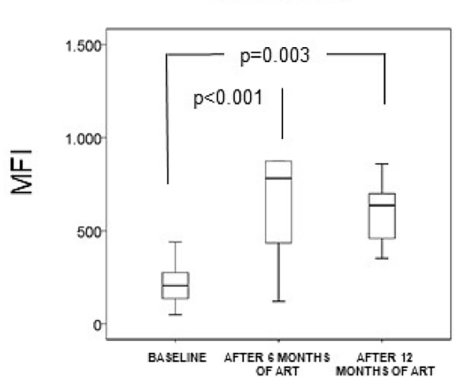

C

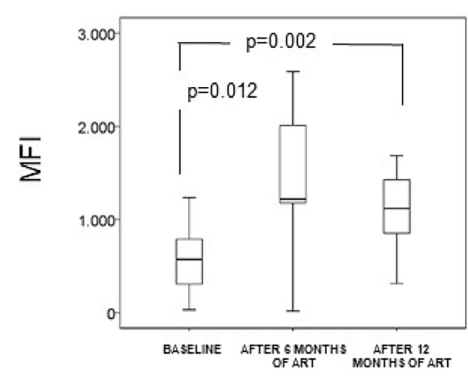

B

PMN-PDL-1+

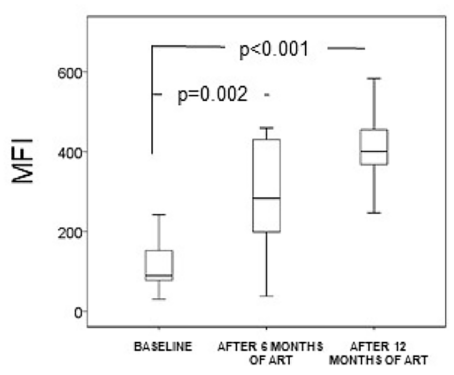

D

PMN-IL-10+

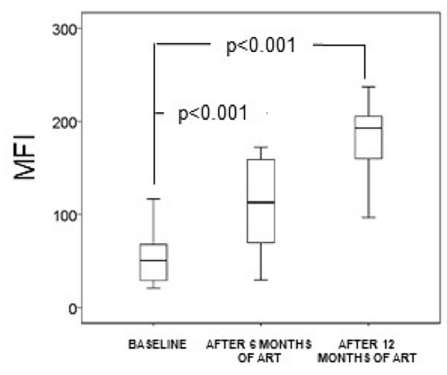

FIGURE 1 | Neutrophils (CD14-CD15+CD16+) (PMN) expression of intracellular IL-6 (A), intracellular arginase 1 (B), membrane PDL-1 (C), and intracellular IL-10 (D) in naiive HIV-infected patients $(n=35)$. They were analyzed at baseline and after 6 and 12 months of antiretroviral treatment. Data are provided as median, interquartile range (boxes) and range of the mean fluorescence intensity (MFI), at baseline and after 6 and 12 months of antiretroviral therapy.

(21 patients) or abacavir and lamivudine (4 patients) combined with integrase inhibitors (18 patients), darunavir/cobicistat (6 patients) or rilpivirine (1 patient). A significantly lower $16 \mathrm{~S}$ rDNA level and serum concentration of IL- 6 and IL-10, although not of I-FABP, was observed in Group 2 patients when compared to group 1 individuals treated for only 12 months; however, serum I-FABP, 16 S rDNA, IL-6 and IL-10 levels were significantly higher than those of healthy controls (Table 2).

The MFI of IL-10 and arginase-1 in peripheral blood PMNs was significantly lower in Group 2 than in Group 1 patients treated for 12 months, although it was higher than in healthy controls (Table 2).

The MFI of BAFF and APRIL in PMNs was significantly lower in chronically infected patients with undetectable HIV at inclusion (Group 2) compared with those treated for 12 months (Group 1). MFI of BAFF and APRIL in PMNs of Group 2 individuals were similar to those observed in healthy controls (Table 2).

B lymphocyte $/ \mathrm{mm}^{3}$ counts were similar in healthy controls, Group 1 patients after 12 months of ART and Group 2 patients. Serum concentrations of $\operatorname{IgG}$ and IgA continued increased in Group 1 patients when compared with healthy controls. In contrast, IgG, IgA and IgM levels were similar in Group 2 HIV-infected patients and healthy controls (Table 2).

\section{DISCUSSION}

Results of this work support the fact of the increased PMN lymphocyte expression of B lymphocyte markers BAFF and APRIL and T lymphocyte molecules PDL-1, IL-10 and arginase-1 in naïve HIV-infected patients. These cell modifications persist after 12 months of ART-induced HIV control, but only those related with $\mathrm{T}$ cell function continue to be present after a prolonged period of HIV undetectability.

PMNs from $\mathrm{HIV}$-infected patients are activated. After recognition of infectious antigens via TLRs, PMNs trigger the synthesis of proinflammatory cytokines and the expression of adhesion molecules, such as CD11b (2). These data explain the increased expression of membrane CD11b and intracytoplasmic IL-6 in the PMNs of the HIV-infected patients that we analyzed in this study, thus supporting previous data (19).

Our data demonstrate that PMNs could influence adaptive immunity. In chronic viral infections, PMNs are able to suppress T-lymphocyte activation (3). The down-regulation of T cells by PMNs has been observed in HIV infection. This could be due to secretion of arginase-1, which depletes L-arginine from the medium and decreases the $\mathrm{T}$ cells viability (14), or through PDL-1 increased expression, which has the ability to interact with $\mathrm{PD}-1$ expressed by $\mathrm{T}$ cells, and induces lymphocyte apoptosis (15). The expression of these molecules, as well as of 
A

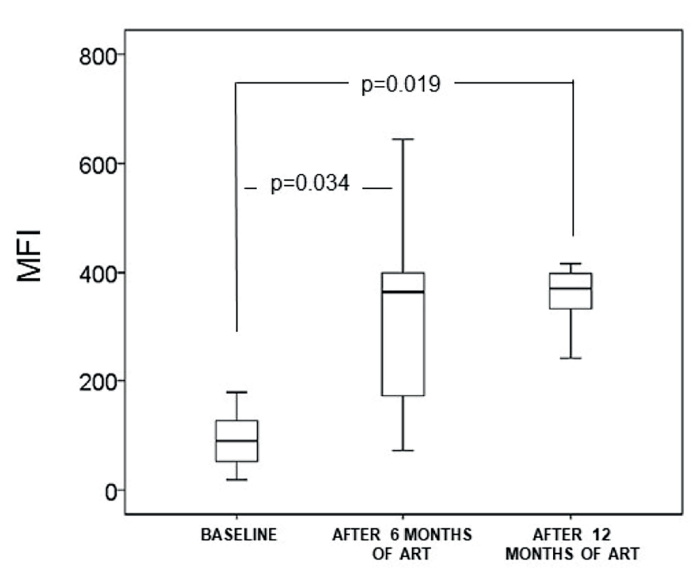

B

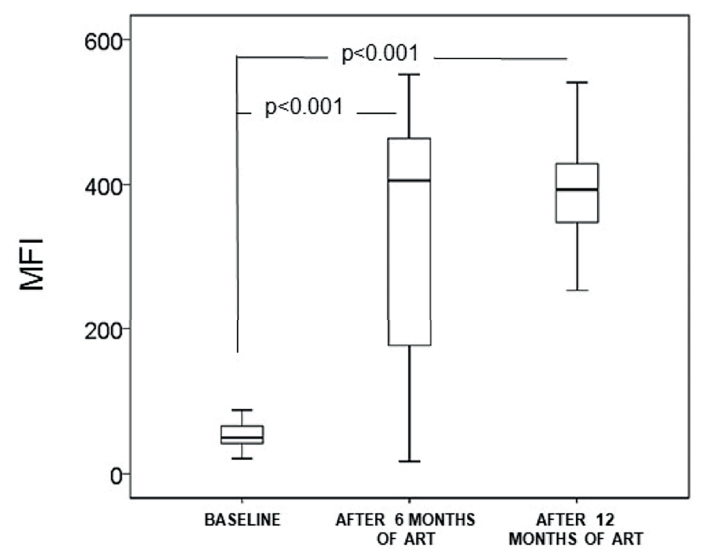

FIGURE 2 | Neutrophils (CD14-CD15+CD16+) (PMN) expression of B cellmodulating markers: intracellular BAFF (A) and intracellular APRIL (B) in naïve infected patients $(n=35)$. They were analyzed at baseline and after 6 and 12 months of antiretroviral treatment. Data are provided as median, interquartile range (boxes) and range of the mean fluorescence intensity (MFI), at baseline and after 6 and 12 months of antiretroviral therapy.

the immunoregulatory IL-10 (20), was significantly increased in the PMNs of untreated HIV-infected patients in our series. This could be, in part, responsible for $\mathrm{T}$ cell dysfunction in HIVinfected individuals.

The IL-10 production by PMNs has been previously discussed in literature. Some authors have concluded that human neutrophils are unable to produce IL-10 (21), while others support the PMN capability to produce it (22-24). Our data are in agreement with those last authors. However, the heterogeneity of human neutrophils has been recognized (25) and thus both interpretations could be correct.

More interestingly, our data imply that PMNs are involved in modulating humoral immunity in these individuals. PMNsderived BAFF and APRIL are implicated in the survival and differentiation of B cells, and in stimulating IgM synthesis and switching to $\operatorname{IgG}$ or $\operatorname{IgA}(8,9)$. We observed an increase in the expression of BAFF and APRIL in untreated HIVinfected patients.

HIV itself, via TLR7/8 (13) interactions, or microbial derived products, via lipopolysaccharide-TLR4 or 16S rDNA-TLR9 interactions (2) could be stimuli implicated in these PMN responses. After ART treatment and controlled viral replication for at least 6 months, microbial translocation-derived molecules could be the main modulators of immune populations. Indeed, and consistent with previous data $(10,11)$, increases in I-FABP and $16 \mathrm{~S}$ rDNA levels persist after ART.

The control of HIV replication is not achieved immediately after beginning ART, continuing the stimuli until undetectable HIV load is obtained. Thus, in our study, the data in which the HIV is eliminated are those patients 6 months after therapy. This could justify that serum IL-6 and IL-10 levels increase and the PMNs activation status and PMNs' expression of T and B cell modulatory molecules continue to be elevated, even to a higher degree in patients after 6 months of ART, reaching a plateau between 6 and 12 months, after undetectable HIV loads have been reached. These findings support that the PMN modulation could be caused, among others, by the persistent intestinal microbial translocation. Viral persistence out of peripheral blood, treatment toxicity and other chronic-related factors may also be implicated in PMN modifications.

On the other hand, bacterial translocation could be influenced by intestinal microbioma, which has been detected to be different in untreated HIV-infected patients, with a depletion of butyrateproducing bacteria (Lachnospiraceae and Ruminococcaceae families) and a higher load of species prone to microbial translation (mainly Enterobacteriaceae) (26). Intestinal dysbiosis may result in increased neutrophil recruitment in intestinal mucosa, as a possible mechanism implicated in microbial translocation (27). Modification of gut microbiota, with a diminution of the number of bacteria with ability to translocate, could decrease the immune activation state in ART-treated HIVinfected individuals, as has been demonstrated by Serrano-Villar et al., using fecal microbial transplantation from healthy individuals (28). These aspects have not been analyzed in our article.

The observed PMN-induced B-cell stimulant response is similar to that detected in the splenic marginal zone (29). It has been theorized that, in the absence of disease, small quantities of intestinal microbial antigens gain access to systemic circulation, activate splenic sinusoidal endothelial cells and induce chemotaxis of PMNs. PMNs are reprogramed to eliminate antigens and provide positive B-cell (BAFF and APRIL expression) signals $(9,30,31)$. In chronic inflammation states, an excessive number of PMNs with these phenotypical characteristics are observed in the spleen, and they can blur the limits of the splenic marginal zone and be observed in peripheral blood (32).

To assess the persistence of these changes in the PMNs, we included a sample of chronic HIV-infected patients treated with ART and presenting HIV undetectability for 101 months (Group 2 ). It is worthy of note that $16 \mathrm{~S}$ rDNA levels were lower in these patients than in those treated for only 12 months (Group 1), 
suggesting a partial improvement in microbial translocation $(10,11)$. Even after more than 8 years of HIV undetectability, the expression of T-cell modulatory molecules (intracellular IL10 and arginase-1), and serum IL-10 levels, continued to be elevated when compared with healthy controls. In contrast, and coincident with the decrease of $16 \mathrm{~S}$ rDNA levels, B cell modulatory molecules levels in Group 2 individuals were significantly lower when compared with Group 1 patients treated for only 12 months. Furthermore, expression values of BAFF and APRIL by PMNs in Group 2 patients were similar to the values expressed by healthy controls. The differences in the $\mathrm{T}$ and B lymphocyte modulation by PMNs, depending on the length of the patients' HIV undetectability (either 1 or 8 years), could be the result of the different sensitivities of PMNderived $\mathrm{T}$ and $\mathrm{B}$ modulation molecules to the levels of $16 \mathrm{~S}$ rDNA.

Serum immunoglobulins levels are influenced by B cell functional state. Indeed, hyperimmunoglobulinemia secondary to polyclonal activation represents an important finding of $\mathrm{B}$ cell dysfunction in HIV-infected patients (33-35). As in our study, other authors have reported that abnormally elevated levels of immunoglobulins persist after 12 months of HIV suppression (36, 37). Asides the T-cell influence on B-cell activation and intrinsic abnormalities of B cells $(33,34)$, the present article supports the additional influence of PMNs on B cell function in HIV-infected individuals and the ability of a prolonged period of HIVreplication control to normalize immunoglobulins concentration.

The possibility of non-specific binding to intracellular proteins could be a potential limitation of this work. However, we assume that the use of FcR blocking reagents, the negative controls to avoid the autofluorescence issue, the strict follow-up of the manufacturers' recommendations and the acquisition of at least 100000 cells in each experiment support the validity of our measurements.

In conclusion, new findings about the pathogenesis of HIV infection are presented: 1) PMNs expression of T-cell regulatory molecules IL-10, PDL-1 and arginase-1 is increased in naïve $\mathrm{HIV}$-infected patients and persists even when a prolonged HIV undetectability period is achieved. 2) PMNs expression of B-cell regulatory molecules BAFF and APRIL is increased in naïve HIV-infected patients and persists during a short period (12 months) of HIV undetectability, but normalizes after a more prolonged period ( 8 years), coinciding with the relative decrease of intestinal microbial translocation. 3) Bacterial translocation persists even after a prolonged period of undetectability. These

\section{REFERENCES}

1. Borregaard N. Neutrophils, From Marrow to Microbes. Immunity (2010) 33:657-70. doi: 10.1016/j.immuni.2010.11.011

2. Kolaczkowska E, Kubes P. Neutrophil Recruitment and Function in Health and Inflammation. Nat Rev Immunol (2013) 13:159-75. doi: 10.1038/nri3399

3. Mantovani A, Cassatella MA, Costantini C, Jaillon S. Neutrophils in the Activation and Regulation of Innate and Adaptive Immunity. Nat Rev Immunol (2011) 11:519-31. doi: 10.1038/nri3024

4. Rotondo R, Bertolotto M, Barisione G, Astigiano S, Mandruzzato S, Ottonello L, et al. Exocytosis of Azurophil and Arginase 1-Containing Granules by Activated Polymorphonuclear Neutrophils Is Required to Inhibit T Lymphocyte Proliferation. J Leukoc Biol (2011) 89:721-7. doi: 10.1189/jlb.1109737 findings suggest a possible contribution of bacterial translocation on $\mathrm{B}$ and $\mathrm{T}$ cell immunomodulation of PMNs.

\section{DATA AVAILABILITY STATEMENT}

The original contributions presented in the study are included in the article/supplementary files, further inquiries can be directed to the corresponding author.

\section{ETHICS STATEMENT}

The studies involving human participants were reviewed and approved by Ethical Research Committee of Hospital Puerta del Mar (Cádiz, Spain). The patients/participants provided their written informed consent to participate in this study.

\section{AUTHOR CONTRIBUTIONS}

Conceived and designed the experiments: MM-C and J-AG-G. Performed the experiments: MM-C, CR, AM-A, CF, SC-S, and J-AG-G. Analyzed the data: MM-C, SC-S, and J-AG-G. Contributed reagents/materials/analysis tools: $\mathrm{MM}-\mathrm{C}, \mathrm{CR}, \mathrm{CF}$; FI-Á; SC-S, and J-AG-G. Wrote the draft: MM-C, SC-S, and J-AG-G. All authors contributed to conception of the study, and critical revision of the manuscript, and saw and approved the final version.

\section{FUNDING}

This work was supported by the Instituto de Salud Carlos III, Acción Estratégica en Salud 2014 (No PI14/01779), Spain. Cofinanced by FEDER (Fondo Europeo de Desarrollo Regional).

\section{SUPPLEMENTARY MATERIAL}

The Supplementary Material for this article can be found online at: https://www.frontiersin.org/articles/10.3389/fimmu.2021.670966/ full\#supplementary-material

5. Zhang X, Majlessi L, Deriaud E, Leclerc C, Lo-Man R. Coactivation of Syk Kinase and MyD88 Adaptor Protein Pathways by Bacteria Promotes Regulatory Properties of Neutrophils. Immunity (2009) 31:761-71. doi: 10.1016/j.immuni.2009.09.016

6. Keir ME, Butte MJ, Freeman GJ, Sharpe AH. PD-1 and Its Ligands in Tolerance and Immunity. Annu Rev Immunol (2008) 26:677-704. doi: 10.1146/annurev.immunol.26.021607.090331

7. Pillay J, Tak T, Kamp VM, Koenderman L. Immune Suppression by Neutrophils and Granulocytic Myeloid-Derived Suppressor Cells: Similarities and Differences. Cell Mol Life Sci (2013) 70:3813-27. doi: 10.1007/s00018-013-1286-4

8. Sakai J, Akkoyunlu M. The Role of BAFF System Molecules in Host Response to Pathogens. Clin Microbiol Rev (2017) 30:991-1014. doi: 10.1128/ CMR.00046-17 
9. Cerutti A, Chen K, Chorny A. Immunoglobulin Responses at the Mucosal Interface. Annu Rev Immunol (2011) 29:273-93. doi: 10.1146/annurevimmunol-031210-101317

10. Brenchley JM, Price DA, Schacker TW, Asher TE, Silvestri G, Rao S, et al. Microbial Translocation Is a Cause of Systemic Immune Activation in Chronic HIV Infection. Nat Med (2006) 12:1365-71. doi: 10.1038/nm1511

11. Jiang W, Lederman MM, Hunt P, Sieg SF, Haley K, Rodriguez B, et al. Plasma Levels of Bacterial DNA Correlate With Immune Activation and the Magnitude of Immune Restoration in Persons With Antiretroviral-Treated HIV Infection. J Infect Dis (2009) 199:1177-85. doi: 10.1086/597476

12. Deeks SG. HIV Infection, Inflammation, Immunosenescence, and Aging. Annu Rev Med (2011) 62:141-55. doi: 10.1146/annurev-med-042909-093756

13. Hensley-McBain T, Klatt NR. The Dual Role of Neutrophils in HIV Infection. Curr HIV/AIDS Rep (2018) 15(1):1-10. doi: 10.1007/s11904-018-0370-7

14. Cloke T, Munder M, Taylor G, Müller I, Kropf P. Characterization of a Novel Population of Low-Density Granulocytes Associated With Disease Severity in HIV-1 Infection. PLoS One (2012) 7:1-7. doi: 10.1371/journal.pone.0048939

15. Bowers NL, Helton ES, Huijbregts RPH, Goepfert PA, Heath SL, Hel Z. Immune Suppression by Neutrophils in HIV-1 Infection: Role of PD-L1/PD-1 Pathway. PLoS Pathog (2014) 10(3):e1003993. doi: 10.1371/journal.ppat.1003993

16. Hunt PW, Sinclair E, Rodriguez B, Shive C, Clagett B, Funderburg N, et al. Gut Epithelial Barrier Dysfunction and Innate Immune Activation Predict Mortality in Treated HIV Infection. J Infect Dis (2014) 210:1228-38. doi: 10.1093/infdis/jiu238

17. Abad-Fernández M, Vallejo A, Hernández-Novoa B, Díaz L, Gutiérrez C, Madrid N, et al. Correlation Between Different Methods to Measure Microbial Translocation and Its Association With Immune Activation in Long-Term Suppressed HIV-1-Infected Individuals. J Acquir Immune Defic Syndr (2013) 64:149-53. doi: 10.1097/QAI.0b013e31829a2f12

18. Tamassia N, Bianchetto-Aguilera F, Arruda-Silva F, Gardiman E, Gasperini S, Calzetti F, et al. Cytokine Production by Human Neutrophils: Revisiting the "Dark Side of the Moon”. Eur J Clin Invest (2018) 48 Suppl 2:e12952. doi: $10.1111 /$ eci.12952

19. Saitoh T, Komano J, Saitoh Y, Misawa T, Takahama M, Kozaki T, et al. Neutrophil Extracellular Traps Mediate a Host Defense Response to Human Immunodeficiency Virus-1. Cell Host Microbe (2012) 12:109-16. doi: 10.1016/ j.chom.2012.05.015

20. Couper KN, Blount DG, Riley EM. IL-10: The Master Regulator of Immunity to Infection. J Immunol (2008) 180:5771-7. doi: 10.4049/jimmunol.180.9.5771

21. Davey MS, Tamassia N, Rossato M, Bazzoni F, Calzetti F, Bruderek K, et al. Failure to Detect Production of IL-10 by Activated Human Neutrophils. Nat Immunol (2011) 12:1017-20. doi: 10.1038/ni.2111

22. De Santo C, Arscott R, Booth S, Karydis I, Jones M, Asher R, et al. Invariant NKT Cells Modulate the Suppressive Activity of IL-10-Secreting Neutrophils Differentiated With Serum Amyloid A. Nat Immunol (2010) 11:1039-46. doi: 10.1038/ni.1942

23. Gómez-Hurtado I, Zapater P, Bellot P, Pascual S, Pérez-Mateo M, Such J, et al. Interleukin-10-Mediated Heme Oxygenase 1-Induced Underlying Mechanism in Inflammatory Down-Regulation by Norfloxacin in Cirrhosis. Hepatology (2011) 53:935-44. doi: 10.1002/hep.24102

24. Lewkowicz N, Mycko MP, Przygodzka P, Ćwiklińska H, Cichalewska M, Matysiak M, et al. Induction of Human IL-10-Producing Neutrophils by LPSStimulated Treg Cells and IL-10. Mucosal Immunol (2016) 9:364-78. doi: 10.1038/mi.2015.66

25. Qi X, Yu Y, Sun R, Huang J, Liu L, Yang Y, et al. Identification and Characterization of Neutrophil Heterogeneity in Sepsis. Crit Care (2021) 25:50. doi: 10.1186/s13054-021-03481-0
26. Vujkovic-Cvijin I, Somsouk M. HIV and the Gut Microbiota: Composition, Consequences, and Avenues for Amelioration. Curr HIV/AIDS Rep (2019) 16:204-13. doi: 10.1007/s11904-019-00441-w

27. Hensley-McBain T, Wu MC, Manuzak JA, Cheu RK, Gustin A, Driscoll CB, et al. Increased Mucosal Neutrophil Survival Is Associated With Altered Microbiota in HIV Infection. PLoS Pathog (2019) 15:e1007672. doi: 10.1371/ journal.ppat.1007672

28. Serrano-Villar S, Talavera-Rodríguez A, Gosalbes MJ, Madrid N, PérezMolina JA, Elliott RJ, et al. Fecal Microbiota Transplantation in HIV: A Pilot Placebo-Controlled Study. Nat Commun (2021) 12:1139. doi: 10.1038/ s41467-021-21472-1

29. Cerutti A, Cols M, Puga I. Marginal Zone B Cells: Virtues of Innate-Like Antibody-Producing Lymphocytes. Nat Rev Immunol (2013) 13:118-32. doi: $10.1038 /$ nri3383

30. Puga I, Cols M, Barra CM, He B, Cassis L, Gentile M, et al. B Cell-Helper Neutrophils Stimulate the Diversification and Production of Immunoglobulin in the Marginal Zone of the Spleen. Nat Immunol (2012) 13:170-80. doi: $10.1038 /$ ni.2194

31. Cerutti A, Puga I, Magri G. The B Cell Helper Side of Neutrophils. J Leukoc Biol (2013) 94:677-82. doi: 10.1189/jlb.1112596

32. Hooper LV, Littman DR, Macpherson AJ. Interactions Between the Microbiota and the Immune System. Science (2012) 336:1268-73. doi: $10.1126 /$ science. 1223490

33. Paiardini M, Müller-Trutwin M. HIV-Associated Chronic Immune Activation. Immunol Rev (2013) 254:78-101. doi: 10.1111/imr.12079

34. Moir S, Fauci AS. B Cells in HIV Infection and Disease. Nat Rev Immunol (2009) 9:235-45. doi: 10.1038/nri2524

35. Brocca-Cofano E, Kuhrt D, Siewe B, Xu C, Haret-Richter GS, Craigo J, et al. Pathogenic Correlates of Simian Immunodeficiency Virus-Associated B Cell Dysfunction. J Virol (2017) 91:e01051-17. doi: 10.1128/JVI.01051-17

36. Jacobson MA, Khayam-Bashi H, Martin JN, Black D, Ng V. Effect of LongTerm Highly Active Antiretroviral Therapy in Restoring HIV-Induced Abnormal B-Lymphocyte Function. J Acquir Immune Defic Syndr (2002) 31:472-7. doi: 10.1097/00126334-200212150-00003

37. Serpa J, Haque D, Valayam J, Breaux K, Rodriguez-Barradas MC. Effect of Combination Antiretroviral Treatment on Total Protein and Calculated Globulin Levels Among HIV-Infected Patients. Int J Infect Dis (2010) 14 Suppl 3:e41-4. doi: 10.1016/j.ijid.2009.10.007

Conflict of Interest: The authors declare that the research was conducted in the absence of any commercial or financial relationships that could be construed as a potential conflict of interest.

Publisher's Note: All claims expressed in this article are solely those of the authors and do not necessarily represent those of their affiliated organizations, or those of the publisher, the editors and the reviewers. Any product that may be evaluated in this article, or claim that may be made by its manufacturer, is not guaranteed or endorsed by the publisher.

Copyright (C) 2021 Márquez-Coello, Ruiz-Sánchez, Martín-Aspas, Fernández Gutiérrez Del Álamo, Illanes-Álvarez, Cuesta-Sancho and Girón-González. This is an open-access article distributed under the terms of the Creative Commons Attribution License (CC BY). The use, distribution or reproduction in other forums is permitted, provided the original author(s) and the copyright owner(s) are credited and that the original publication in this journal is cited, in accordance with accepted academic practice. No use, distribution or reproduction is permitted which does not comply with these terms. 